\title{
Segmentos de vertente e atributos do solo de uma toposseqüência na região de Manicoré, $\mathbf{A} \mathbf{M}^{1}$
}

\author{
Slope segments and soil properties of a topossenquence in the Manicoré region, AM
}

\author{
Milton César Costa Campos ${ }^{2 *}$, Mateus Rosas Ribeiro ${ }^{3}$, Valdomiro Severino de Souza Júnior ${ }^{3}$, Mateus Rosas \\ Ribeiro Filho ${ }^{3}$ e Edivan Uchôa Cavalcanti da Costa ${ }^{4}$
}

\begin{abstract}
Resumo - As variações dos atributos do solo ocorrem em função de vários fatores tais como: clima, material de origem, vegetação e, especialmente do relevo, que regula o escoamento superficial e a drenagem, e tem influência no tempo de exposição dos materiais à ação do intemperismo. O objetivo deste trabalho foi estudar a influência dos diferentes segmentos de vertente nos atributos do solo em uma toposseqüência na região de Manicoré, AM. Foi estabelecido um caminhamento de $3.000 \mathrm{~m}$ a partir do "espigão" da vertente no sentido do caimento mais suave do relevo e os segmentos da vertente foram determinados com base na "quebra" de declive do terreno. Foram coletadas 20 amostras nos diferentes segmentos de vertentes identificados: topo alto: 0,0-0,19 e 0,37-0,60 m; meia encosta: 0,0-0,28 e 0,60-0,80 m; sopé de transporte: $0,0-0,15$ e 0,42-0,71 m e topo baixo: 0,0-0,15 e 0,20-0,40 m. O critério de escolha das profundidades foi à coincidência com os horizontes diagnósticos superficiais e subsuperficiais determinados na descrição morfológica dos perfis. Foram realizadas análises físicas de textura, densidades do solo e das partículas, porosidade total e condutividade hidráulica, Nas análises químicas foram determinados pH, em água e em $\mathrm{KCl}, \mathrm{Ca}, \mathrm{Mg}, \mathrm{K}$, e $\mathrm{Al}$ trocáveis, $\mathrm{P}$ disponível, $\mathrm{H}+\mathrm{Al}$ e $\mathrm{C}$ orgânico. Com o uso das técnicas estatísticas multivariadas foi possível distinguir diferentes ambientes geomórficos independentes dos segmentos de vertente identificados. As variações do relevo favoreceram a presença de solos distróficos no topo alto e solos eutróficos no sopé de transporte.
\end{abstract}

Palavras-chave - Formas do relevo. Manicoré. Pedogênese.

\begin{abstract}
Variations of soil properties are consequence of several factors such as: climate, parent material, vegetation and, particularly relief, which controls runoff and drainage, and has influence in the exposure of parent materials to the weathering action. The objective of this work was to study the influence of the slope segments on soil attributes of a topossenquence in the Manicoré region, AM. Twenty samples were collected in each of the several slope segments identified along a $3 \mathrm{~km}$ transect, from the top and toward the lower part of the topography. The slope segments and the sampling depths were: high top (0.0-0.19 and 0.37-0.60 m), medium slope (0.0-0.28 and 0.60-0.80 m); lower slope (0.0-0.15 and 0.42-0.71 m) and low top (0.0-0.15 and $0.20-0.40 \mathrm{~m})$. The sampling depths were coincident with the surface and subsurface diagnostic horizons defined in the profile morphological description. Physical analysis involved particle size distribution, soil and particle density, total porosity and saturated hydraulic conductivity. In the chemical analysis $\mathrm{pH}$ in water and $\mathrm{KCl}$, exchangeable cations, exchangeable $\mathrm{Al}$, available $\mathrm{P}, \mathrm{H}+\mathrm{Al}$, organic carbon were determined. The use of multivariate statistic techniques enabled the distinction of different soil geomorphic environments independent of the slope segments identifications. The relief variations contributed to the presence of dystrophic soils in the high top and eutrophic soils in the lower slope.
\end{abstract}

Key words - Landscape forms. Manicoré. Pedogenesis.

\footnotetext{
* Autor para correspondência

${ }^{1}$ Recebido para publicação em 10/02/2010; aprovado em 02/09/2010

Parte da Tese do primeiro autor apresentada no Programa de Pós-Graduação em Ciências do Solo da UFRPE, financiada pelo CNPq e FAPEAM

${ }^{2}$ Colegiado de Agronomia, IEAA/UFAM, Humaitá-AM, Brasil, mcesarsolos@gmail.com

${ }_{3}^{3}$ Departamento de Agronomia, UFRPE, Recife-PE, Brasil, mrosas@depa.ufrpe.br, vsouzajr@yahoo.com, mateusrf@depa.ufrpe.br

${ }^{4}$ Programa de Pós-Graduação em Ciências do Solo, UFRPE, bolsista do CNPq, Recife-PE, Brasil, edivanuchoa@hotmail.com
} 


\section{Introdução}

As diferentes formas da paisagem expressas pelo relevo, em várias escalas, provocam variações nos atributos do solo em magnitudes diferenciadas, dependentes de um local específico da paisagem (IPPOLITI et al., 2005). Segundo Campos et al. (2007), fatores como hidrologia, litologia e as condições climáticas também são responsáveis por essa variabilidade, principalmente quando associados aos acontecimentos geológicos e geomorfológicos históricos, que influenciam a dinâmica de ocorrência e o grau de evolução dos solos nas paisagens.

Dessa forma, estudar o comportamento dos solos, considerando os diferentes segmentos das vertentes é uma boa maneira de entender a sua distribuição espacial, pois permite compreender os processos que envolvem o movimento e armazenamento de água dentro do perfil do solo e, ainda, sua relação com o transporte e a deposição de sedimentos, de maneira a provocar variabilidade espacial nos seus atributos (PACHEPSKY et al., 2001). Vale salientar que de acordo com Park e Burt (2002), apesar de muitos fatores causarem variação nos atributos do solo, os aspectos topográficos são os mais importantes indicadores da variação das propriedades do solo, em nível local.

Nesse sentido vários autores preocuparam-se em investigar a influência do relevo na variação espacial dos atributos do solo, dentre estes os estudos desenvolvidos por Cunha et al. (2005) que investigaram as relações solo-geomorfologia em áreas de transição arenito-basalto, relacionando a variabilidade espacial dos atributos do solo aos diferentes compartimentos de relevo.

Para Carrée Mcbratney(2005), quanto mais distintos os ambientes geomórficos, mais importantes tornam-se os estudos que relacionam o padrão de distribuição espacial dos atributos do solo e suas relações de dependência com a disposição do relevo. Nesse sentido, vários estudos foram desenvolvidos buscando esclarecer e constatar as relações entre os atributos do solo e as condições da paisagem (BARTHOLD et al., 2008; HENDERSON et al., 2005).

A análise estatística multivariada surgiu como importante ferramenta na obtenção de maior quantidade de informação que dificilmente seria gerada com o uso de métodos univariados. Assim, o uso de técnicas multivariadas permitiu que fenômenos dependentes de múltiplas variáveis pudessem ser analisados, favorecendo assim um entendimento maior do complexo, já que não basta conhecer as variáveis isoladas, mas conhecê-las na sua totalidade, pois as informações são fornecidas pelo conjunto e não individualmente (GROBE, 2005). Por outro lado, a utilização do sistema de classificação numérica e hierárquica revela os vários níveis da similaridade e permite visualizar um número variável de agrupamentos; com isso a variabilidade é minimizada dentro de um grupo e maximizada entre os grupos, a fim de produzir grupos relativamente similares (YEMEFACK et al., 2005).

Apesar da importância do tema, em ambiente amazônico, poucos são os trabalhos que destacam essas relações, pois as informações sobre a distribuição e o comportamento dos solos na região baseiamse principalmente, em levantamentos generalizados (CAMPOS, 2009). Dessa forma o objetivo deste trabalho foi investigar as influências dos diferentes segmentos de vertente nos atributos do solo em uma toposseqüência sobre granito na região de Manicoré, AM.

\section{Material e métodos}

\section{O meio físico}

A área de estudo localiza-se nas imediações da comunidade de Santo Antônio de Matupi, às margens da BR 319 - rodovia Transamazônica, Município de Manicoré, sentido Humaitá-Apuí-AM, situada nas coordenadas geográficas de 07059'77,1" S e 061³9'51,2” W. A configuração do relevo é marcada pela presença de platôs nas partes mais elevadas, que exibem superfícies topográficas planas, sendo a zona de borda marcada por colinas e cristas alinhadas, enquanto que as áreas mais baixas constituem uma superfície pediplanada, localmente interrompida por colinas de topo plano (CPRM, 2001).

Com relação à geologia, a área de estudo foi localizada sobre Granitos Rondonianos que se caracterizam pela presença de muscovita, biotita, adamelitos e granodioritos, de origem intrusiva cratogênica, em forma de "stocks" e batólitos (BRASIL, 1978). De acordo com o ZEE (2008), os solos presentes na região são os Latossolos Amarelos e Vermelho-Amarelos. A vegetação característica desta região é a Floresta Tropical Densa constituída por árvores adensadas e multiestratificadas entre 20 a 50 metros de altura. O clima da região, segundo a classificação de Köppen, é do tipo tropical chuvoso (chuvas do tipo monção), apresentando um período seco de pequena duração (Am), temperaturas variando entre 25 e $27{ }^{\circ} \mathrm{C}$ e precipitação média anual de $2.500 \mathrm{~mm}$, com período chuvoso iniciando em outubro e prolongando-se até junho e umidade relativa do ar entre 85 e $90 \%$.

\section{Métodos de trabalho de campo, laboratório e escritório}

Foi estabelecido um caminhamento, seguindo o "espigão" da vertente no sentido do caimento mais suave do declive, partindo-se do topo até a área de sopé. Ao longo do caminhamento foram realizadas mensurações das 
altitudes para a confecção do perfil altimétrico. Com base no modelo de Dalrymple et al. (1968), foram identificados os segmentos da vertente, com base, principalmente, na variação da declividade do terreno (FIG. 1).

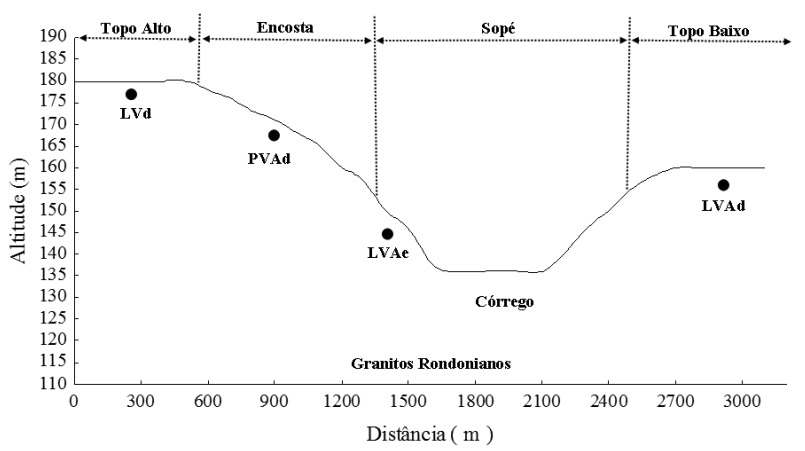

Figura 1 - Perfil topográfico, superfícies geomórficas, segmentos de vertente e suas respectivas classes de solos e substrato rochoso em Santo Antônio do Matupi, Manicoré, AM

Foram abertas trincheiras nos segmentos de vertente mapeados para caracterização morfológica, física e química. Foram realizadas a identificação dos horizontes e a descrição morfológica seguida de coleta de amostras dos horizontes dos solos. Após esta etapa os solos foram classificados segundo critérios estabelecidos pelo Sistema Brasileiro de Classificação de Solo (SiBCS).

Foram coletadas 20 amostras nos diferentes segmentos de vertentes identificados: topo alto: 0,0-0,19 e 0,37-0,60 m; meia encosta: 0,0-0,28 e 0,60-0,80 m; sopé de transporte: $0,0-0,15$ e $0,42-0,71 \mathrm{~m}$ e topo baixo: $0,0-0,15$ e $0,20-0,40 \mathrm{~m}$. O critério de escolha das profundidades foi a coincidência com os horizontes diagnósticos superficiais e subsuperficiais.

A análise granulométrica foi realizada pelo método da pipeta, utilizando uma solução de $\mathrm{NaOH}$ 0,1 N como dispersante químico, e agitação mecânica em aparato de alta rotação por 10 minutos. A fração argila foi separada por sedimentação, as areias grossa e fina por tamisação e o silte calculado por diferença, segundo metodologia da Embrapa (1997).

A densidade do solo foi obtida pelo método do anel volumétrico e a densidade de partícula pelo método do balão volumétrico conforme metodologia da Embrapa (1997). A porosidade total foi calculada a partir dos dados obtidos das densidades do solo e de partícula, empregando-se a seguinte expressão:

$$
P t=100\left(1-\frac{D s}{D p}\right)
$$

sendo $\mathrm{Pt}=$ porosidade total; $\mathrm{Ds}=$ densidade do solo; $\mathrm{Dp}=$ densidade de partícula

A condutividade hidráulica saturada dos solos foi determinada em amostras deformadas (TFSA), conforme metodologia proposta pela Embrapa (1997).

Cálcio, magnésio e alumínio trocáveis, o potássio, sódio e fósforo disponível e a acidez potencial $(\mathrm{H}+\mathrm{Al})$ foram determinados utilizando-se metodologia proposta pela Embrapa (1997). Com base nos resultados das análises químicas, foram calculadas a soma de bases (SB), a capacidade de troca catiônica (CTC), a saturação por bases (V\%) e alumínio. $\mathrm{O} \mathrm{pH}$ foi determinado potenciometricamente utilizando-se relação 1:2,5 de solo: em água e $\mathrm{KCl}$ (EMBRAPA, 1997). O carbono orgânico total foi determinado pelo método de oxidação via úmida, com aquecimento externo (YEOMANS; BREMNER, 1988), e a matéria orgânica foi estimada com base no carbono orgânico total.

Com os resultados das amostras coletadas nos segmentos de vertente, foram realizadas análises de estatística descritivas, com a utilização de boxplot para apresentar as medidas de tendência central, de dispersão, dados discrepantes e extremos.

Foram aplicadas às amostras de solos dos segmentos de vertente, dois métodos estatísticos multivariados, visando classificar os acessos em grupos: análise de agrupamentos hierárquica e análise de componentes principais (ACP). Todas as análises multivariadas foram realizadas após a padronização das variáveis $(\mu=0, \sigma=1)$. A análise de agrupamentos hierárquica foi realizada calculando-se a distância euclidiana entre os acessos, para o conjunto das vinte e duas variáveis, e utilizando o algoritmo de Ward para a obtenção dos agrupamentos de acessos similares. O resultado da análise foi apresentado em forma gráfica (dendrograma) que auxiliou na identificação dos agrupamentos dos acessos, baseando-se na técnica empírica descrita por Hair et al. (2005).

A ACP permite condensar a maior quantidade da informação original contida em $p$ variáveis $(p=22$, neste estudo) em duas variáveis latentes ortogonais denominadas componentes principais, que são combinações lineares das variáveis originais criadas com os dois maiores autovalores da matriz de covariância dos dados, para Gomes et al. (2004) na maioria dos estudos em Ciência do Solo usamse apenas os dois primeiros eixos, considerados suficientes para explicar os dados e pela facilidade de interpretação de um gráfico em duas dimensões. Desta forma, o conjunto inicial de vinte duas variáveis passou a ser caracterizado por duas novas variáveis latentes, o que possibilitou sua localização em figuras bidimensionais (ordenação dos acessos por componentes principais). Todas as análises estatísticas foram processadas com auxilio do programa estatístico Statistica 7.0 (STATITICA, 2005). 


\section{Resultados e discussão}

Foram realizadas análises estatísticas, com as amostras laterais, considerando apenas os segmentos de vertente como fontes de variação. Nas Figuras 2 e 3 são apresentados os resultados referentes aos atributos físicos.
Em ambos os horizontes diagnósticos os teores de areia foram mais elevados na encosta e no sopé de transporte quando comparados às posições de topo (FIG. 2). Foi verificado também maior variabilidade dos dados, com maiores intervalos de confiança. Este resultado é atribuído às formas do relevo destes

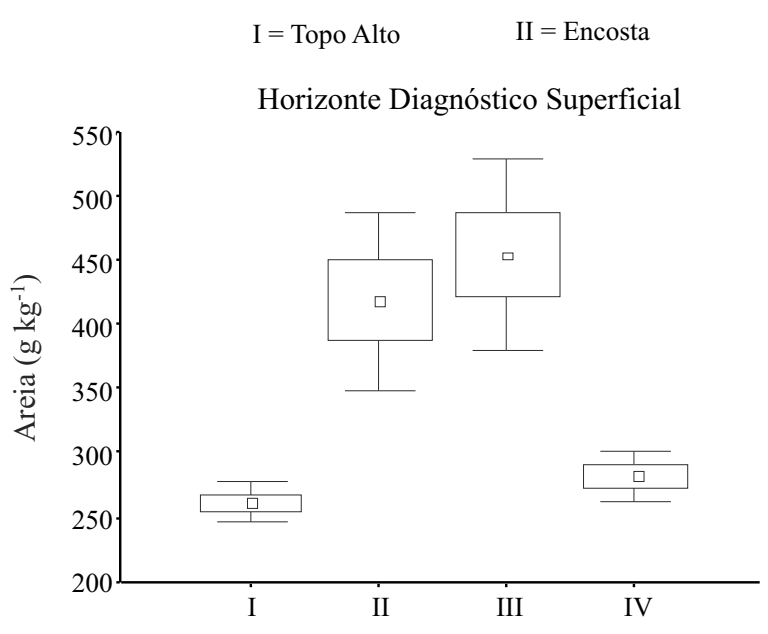

$\mathrm{IV}=$ Topo Baixo
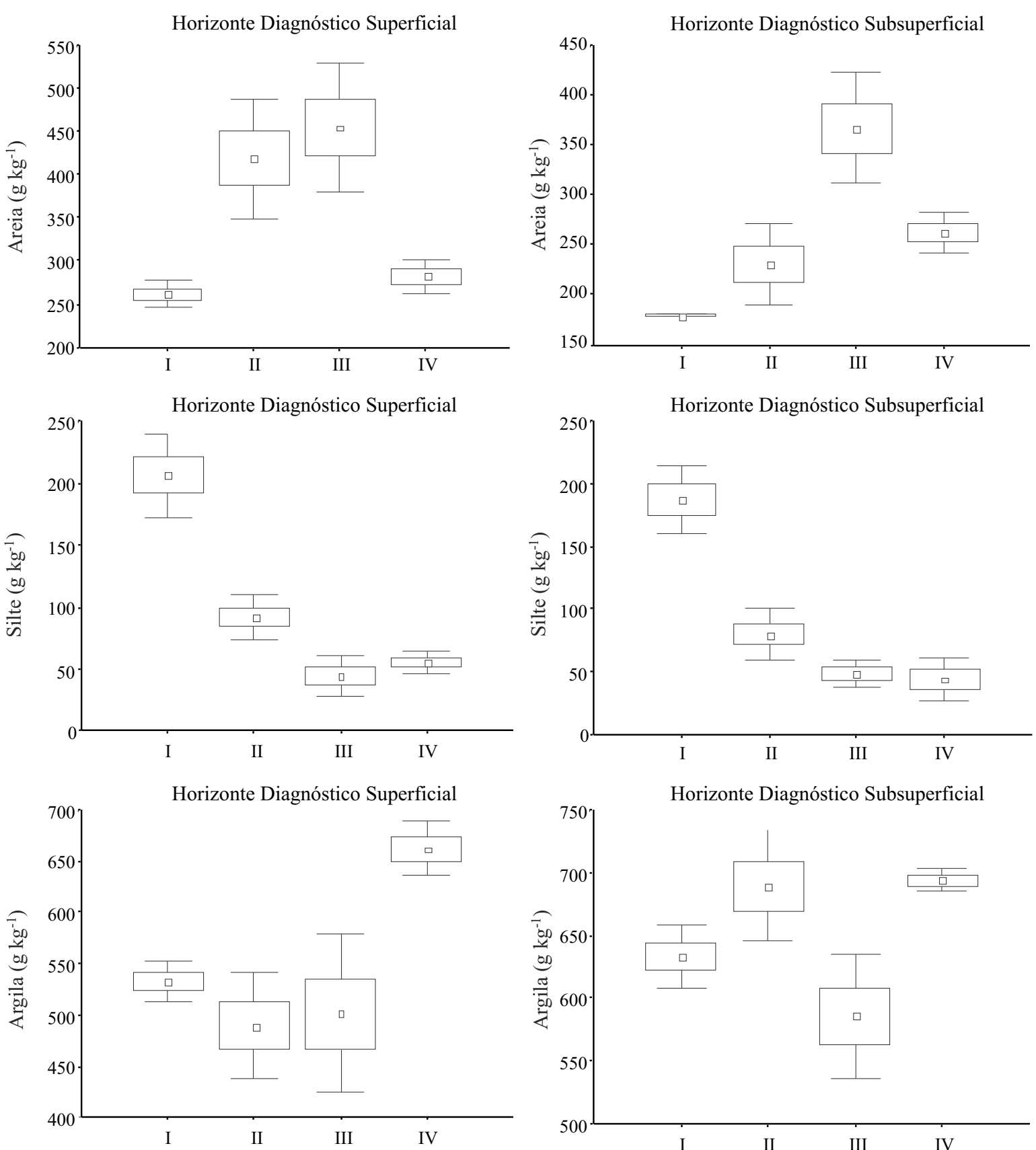

Horizonte Diagnóstico Subsuperficial

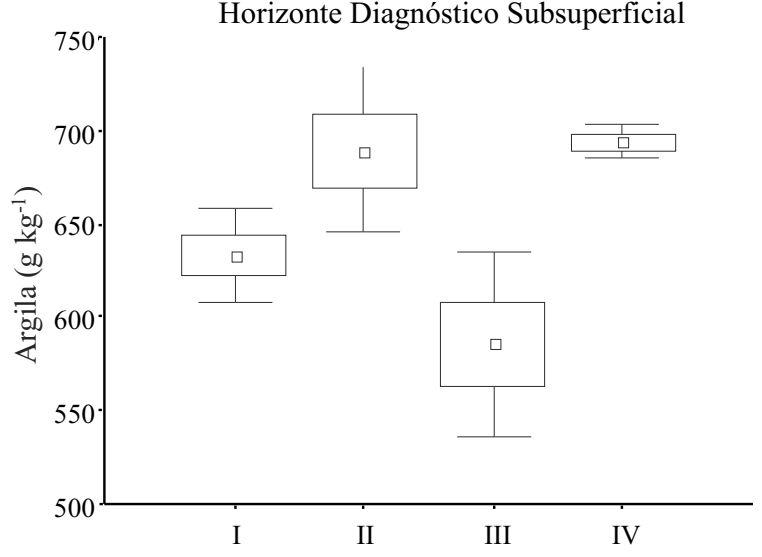

Segmentos de Vertente

Segmentos de Vertente

Figura 2 - Médias, com respectivos intervalos de confiança, dos teores de areia, silte e argila do solo por segmentos de vertente 


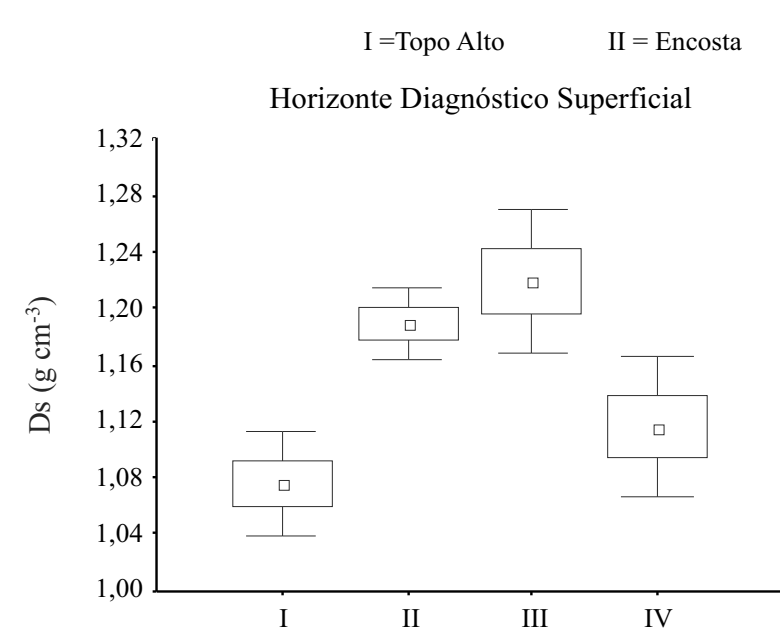

$$
\text { III = Sopé de Transporte } \quad \text { IV = Topo Baixo }
$$
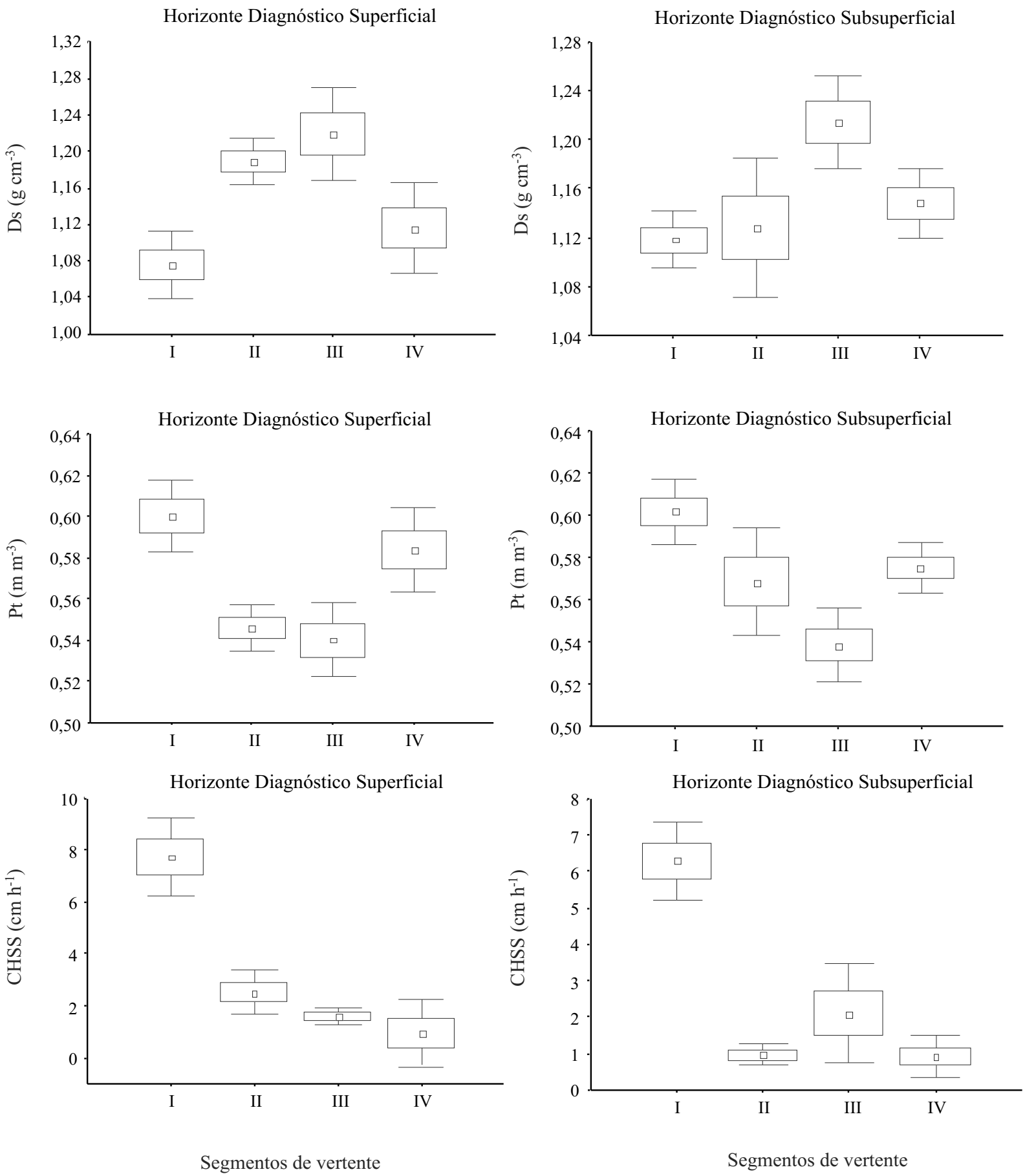

Figura 3 - Médias, com respectivos intervalos de confiança, da densidade do solo (Ds), porosidade total (Pt) e condutividade hidráulica do solo saturado por segmentos de vertente

ambientes (encosta e sopé de transporte), que favorecem o carreamento de partículas mais finas e conseqüente concentração de frações mais grosseiras, dando maior instabilidade ao ambiente, conforme destaca VidalTorrado et al. (1999).
No caso da fração argila o comportamento foi inverso, exibindo maiores concentrações nas posições de topo em relação à encosta e sopé de transporte (FIG. 2), entretanto as maiores variabilidade dos dados foram observadas também nos segmentos de sopé e 
encosta, fato que se deve a natureza do material de origem associado às formas do relevo mais estável, que não favorece o transporte de material para outros compartimentos da paisagem conforme destaca Barthold et al. (2008).

Os resultados da densidade do solo foram mais elevados no sopé de transporte do que nas outras unidades da paisagem, possivelmente em conseqüência da maior instabilidade desse ambiente geomórfico (FIG. 3). Nos topo alto e baixo, as condições de maior intemperismo e maiores teores de matéria orgânica favorecem uma maior agregação e estabilidade estrutural do solo, o que reflete, por sua vez, na maior porosidade total (FIG. 3). Em relação à variabilidade geral dos valores de densidade do solo verificou-se que para ambos os horizontes diagnóstico o ambiente geomórfico de sopé de transporte foi aquele que apresentou maiores intervalos de confiança, indicando relações intimas entre o comportamento deste variável e as condições da paisagem (HENDERSON et al., 2005).

A condutividade hidráulica do solo saturado apresentou também valores maiores no segmento de topo alto da paisagem (FIG. 3). Segundo Sobieraj et al., (2002), estes resultados são influenciados pelas condições de maior estabilidade do terreno, conteúdo de matéria orgânica e agregação do solo, que favorecem maior capacidade de infiltração. Por outro lado os maiores intervalos de confiança, que por sua vez, evidenciam maior variabilidade da condutividade hidráulica foi verificada no topo alto de ambos os horizontes diagnósticos e no sopé de transporte dos horizontes diagnósticos subsuperficiais, estes resultados possivelmente estão associados às caracteristicas da própria variável.

Os valores do $\mathrm{pH}$ foram semelhantes em ambos os horizontes diagnósticos, apresentando valores com tendência de decréscimo partindo do topo alto em direção ao segmento de topo baixo e com maior variabilidade espacial no horizonte diagnóstico subsuperficial em todos os ambientes geomórficos (FIG. 4). De acordo com Pachepsky et al. (2001) essa maior variabilidade dos dados deve-se ao movimento anisotrópico da água no interior do solo, influenciados pelas condições topográficas, localização no terreno e tipo de horizonte diagnóstico subsuperficial (B).

Os teores de $\mathrm{Al}^{3+}$ trocável apresentaram comportamento oposto ao $\mathrm{pH}$ em água, com valores crescente no sentido do topo baixo (FIG. 4). Apesar dessa expressão do $\mathrm{Al}^{3+}$ trocável maior no topo baixo, verificou-se que a maior variabilidade ocorreu no topo alto dos horizontes diagnósticos superficiais este fato deve-se possivelmente a posição mais estável deste ambiente que condiciona maior lixiviação de cátions trocáveis e variação nos teores deste componente (CARRÉ; MCBRATNEY, 2005).

Foi observado que a soma de bases (SB) apresentou comportamento diferente nos horizontes diagnósticos: em superfície os valores foram bastante semelhantes no topo alto, encosta e sopé de transporte, com valores mais baixos no topo baixo. Em subsuperfície o topo alto apresentou menores valores médios quando comparado aos demais segmentos de vertente, já o sopé de transporte apresentou o maior valor médio. Este comportamento é produto das contribuições oriundos das áreas adjacente, pois este é um ambiente geomórfico de deposição de materiais, conforme sugere Park e Burt (2002) em que destacam os aspectos topográficos como importantes indicadores de variação das propriedades do solo, em nível local.

A capacidade de troca catiônica (CTC) em ambas as profundidades apresentou comportamento semelhante, com valores mais elevados nos topos altos e baixos, quando comparados aos demais segmentos de vertente, estes resultados mais elevados nestes dois segmentos de vertente devem-se às contribuições dada pela acidez potencial que apresentou valores mais expressivos nestes locais (FIG. 5).

A saturação de bases (V\%) foi maior no segmento de sopé de transporte, seguido pela encosta, topo baixo e topo alto. Ainda em relação ao $\mathrm{V} \%$ verificou-se que o solo do segmento sopé de transporte é eutrófico, enquanto os demais segmentos (encosta, topo alto e topo baixo) são distróficos. A saturação por alumínio $(\mathrm{m} \%)$ nos dois horizontes diagnósticos tem comportamento inverso ao dos valores do $\mathrm{V} \%$, sendo maior no topo baixo, confirmando valores obtidos por Nunes et al. (2001) que estudaram a relação solopaisagem-material de origem em solos de Minas Gerais.

Os dendrogramas obtidos pela análise de agrupamento para os horizontes superficiais e subsuperficiais são apresentados na Figura 6. Nesta análise as amostras (pontos de amostragem de solo) foram agrupadas com base no seu grau de semelhança, com o objetivo de classificá-las em grupos similares.

Para os horizontes diagnósticos superficiais, foi admitido um corte na distância de ligação de 5,0 (FIG. 6), que permitiu uma divisão clara de dois grupos. Isto indica que, o uso conjunto dos atributos físicos e químicos permitiu ordenar os dados em apenas dois grupos dos segmentos de vertente. O G1, englobando os dados localizados no topo baixo, sopé de transporte e encosta da paisagem, enquanto que o G2 agrupando os dados a posição de topo alto, evidenciando assim, que os segmentos de vertente topo baixo, encosta e sopé de transporte apresentaram semelhanças em seus atributos diferindo do topo alto. 
$\mathrm{I}=$ Topo Alto

II $=$ Encosta

Horizonte Diagnóstico Superficial

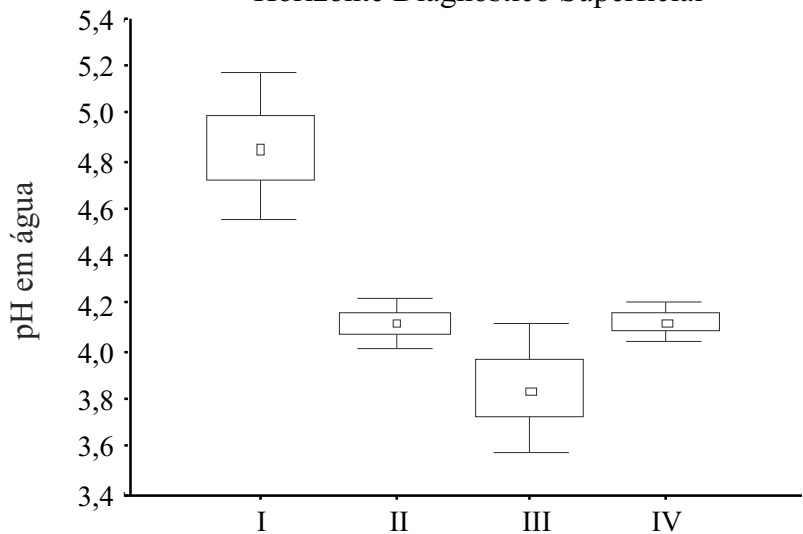

Horizonte Diagnóstico Superficial
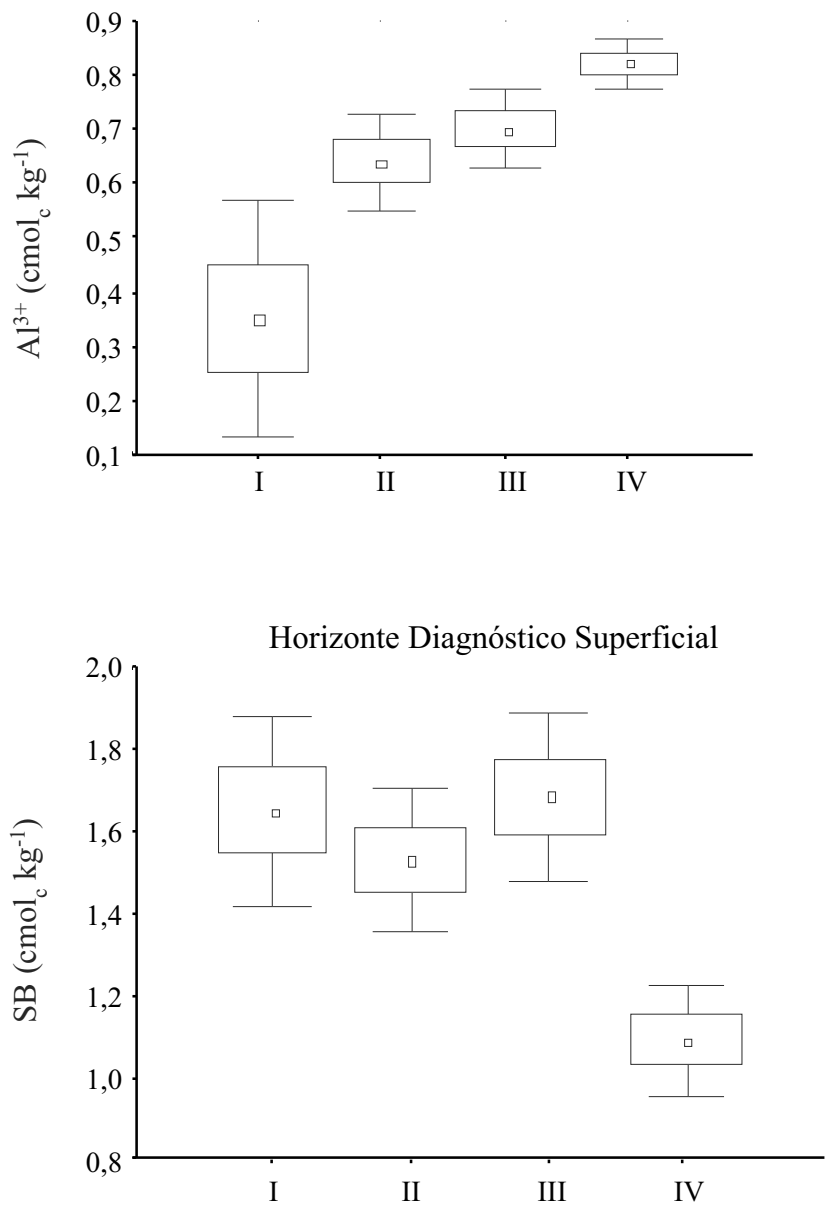

Segmentos de Vertente
III = Sopé de Transporte $\quad$ IV = Topo Baixo

Horizonte Diagnóstico Subsuperficial

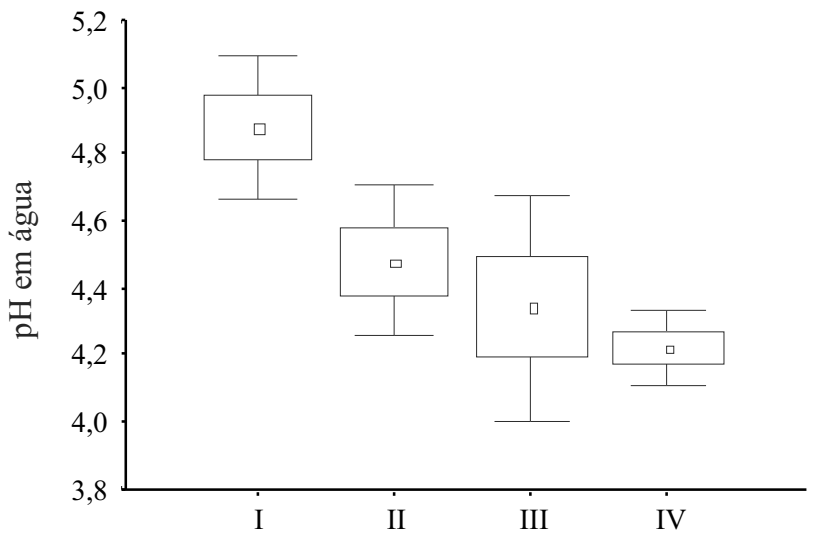

Horizonte Diagnóstico Subsuperficial

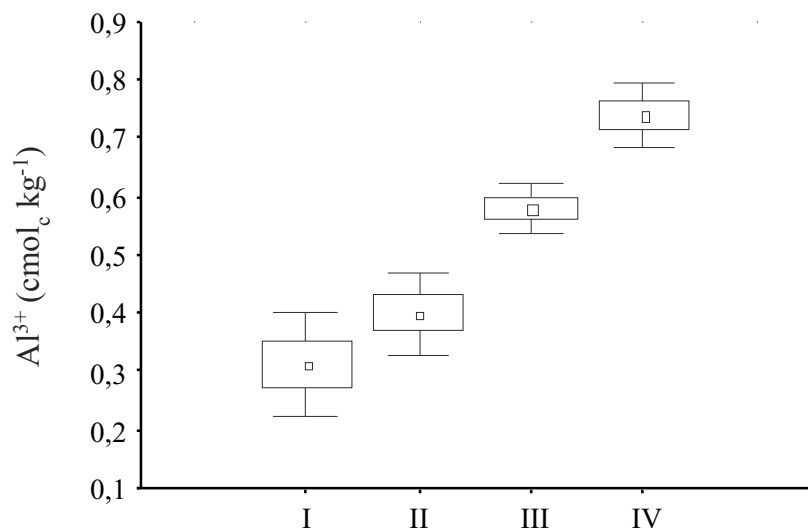

Horizonte Diagnóstico Subsuperficial

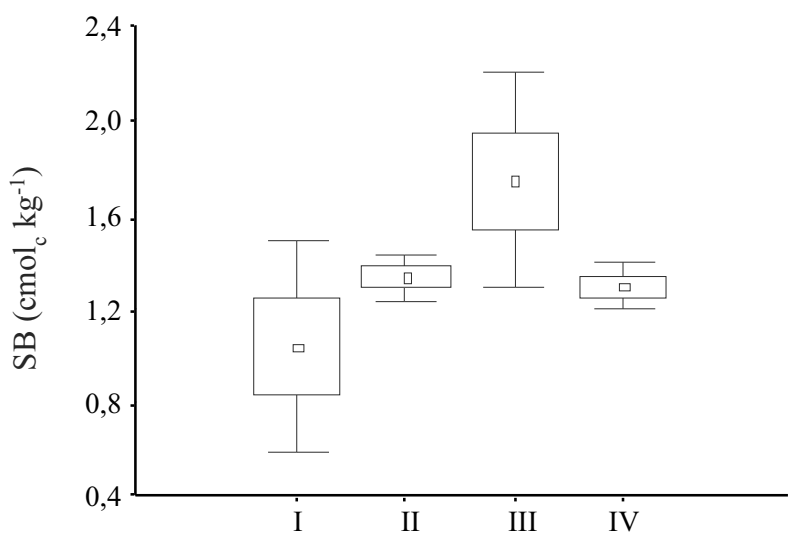

Segmentos de Vertente

Figura 4 - Médias, com respectivos intervalos de confiança, do $\mathrm{pH}$ em $\mathrm{H}_{2} \mathrm{O}, \mathrm{Al}^{3+}$ e $\mathrm{SB}$ do solo por segmentos de vertente 
III = Sopé de Transporte $\quad$ IV = Topo Baixo

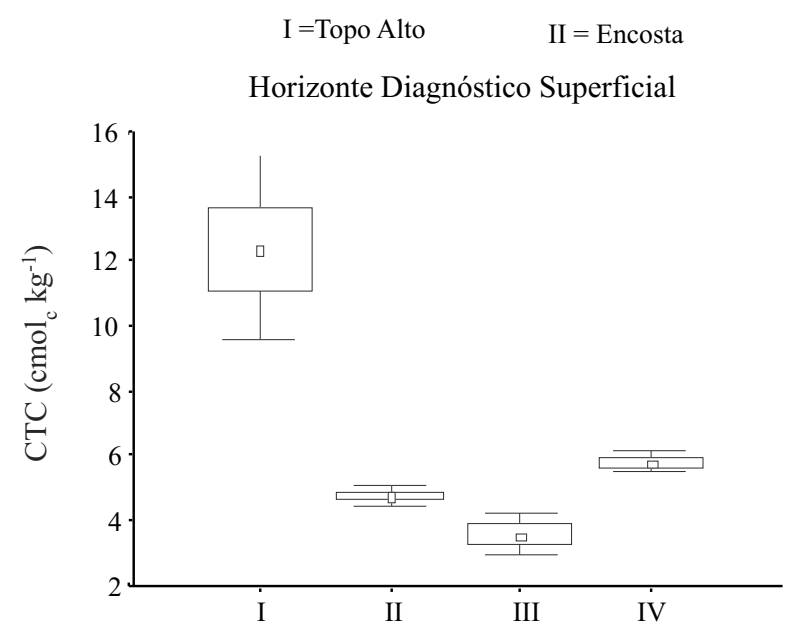

Horizonte Diagnóstico Superficial
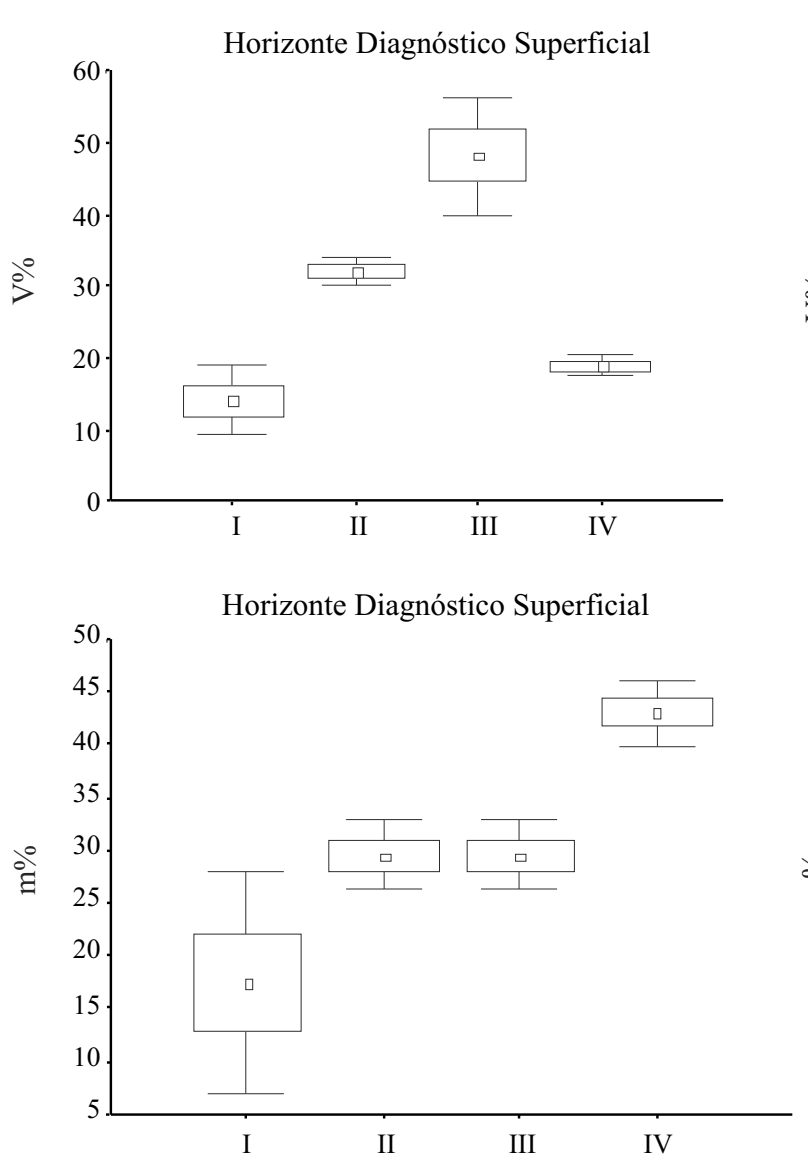

Segmentos de Vertente

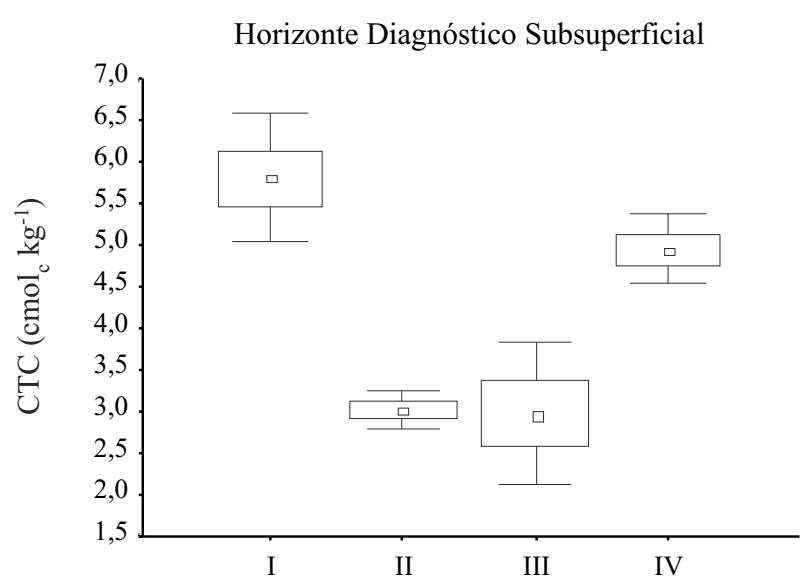

Horizonte Diagnóstico Subsuperficial

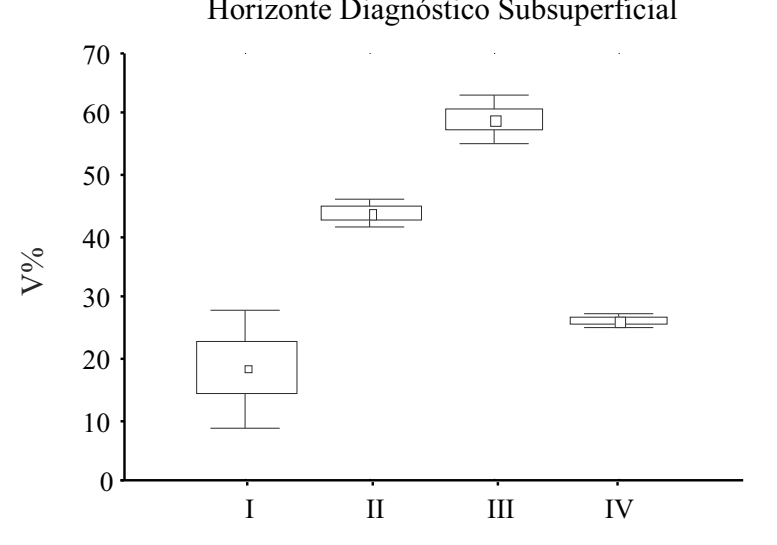

Horizonte Diagnóstico Subsuperficial

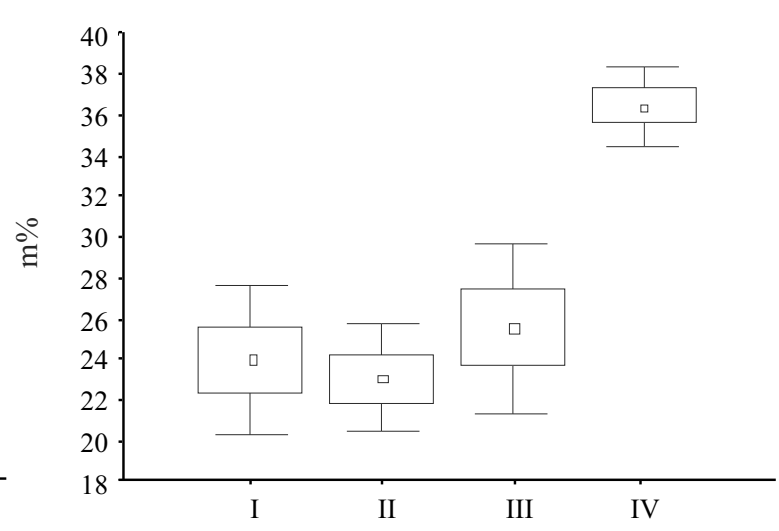

Segmentos de Vertente

Figura 5 - Médias, com respectivos intervalos de confiança da CTC, V\% e m\% do solo por segmentos de vertente

Esta informação corrobora com a afirmativa de Yemefack et al. (2005) que esta técnica permite agrupar variáveis com características semelhantes e com aumento de variabilidade entre os agrupamentos formados. Para
Cunha et al. (2005) os resultados acima ainda podem ser explicados pelo fato de um grupo de solos (G1) ocuparem as posições mais instáveis da paisagem e por serem horizontes superficiais o que permite este resultado. 

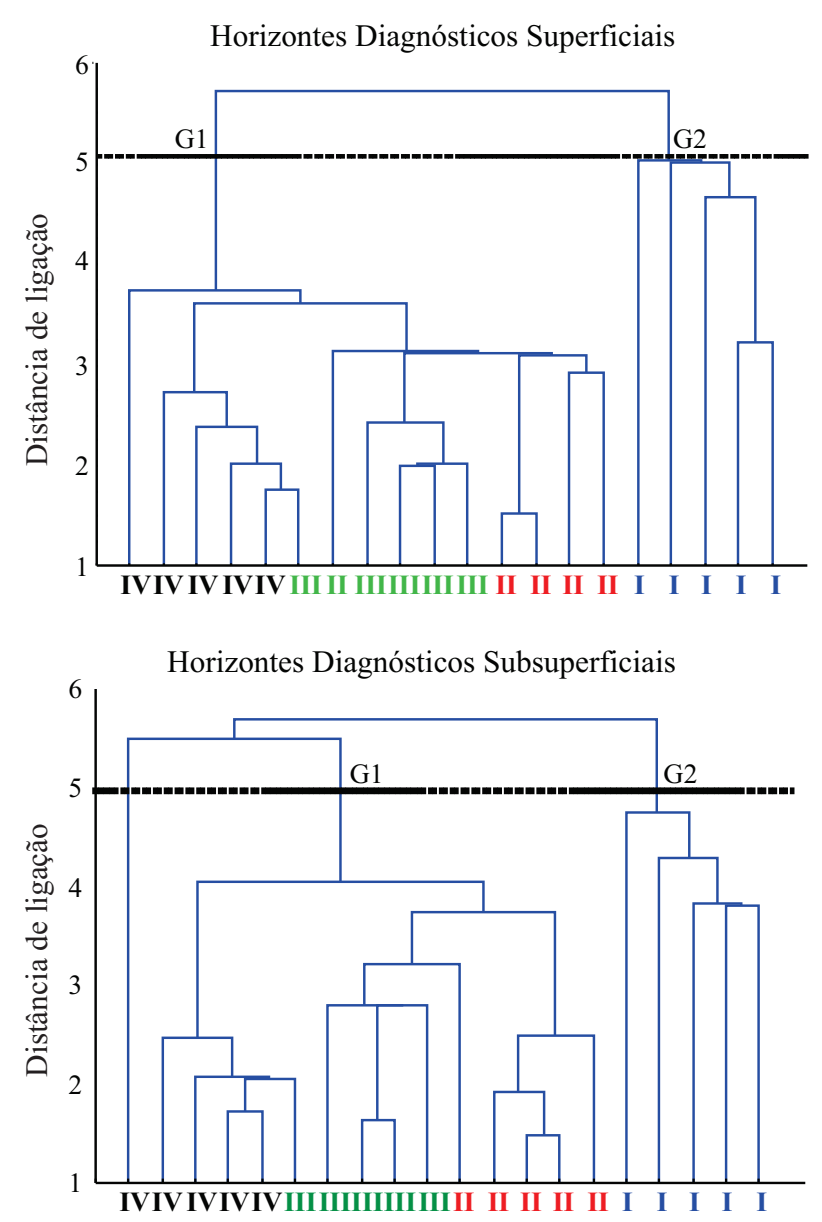

$\mathbf{I}=$ topo alto $\quad \mathbf{I I}=$ encosta $\quad \mathbf{I I I}=$ sopé de transporte $\quad \mathbf{I V}=$ topo baixo

Figura 6 - Dendrograma das interrrelações dos segmentos de vertente, referente ao conjunto de parâmetros físicos (areia, silte, argila, $\mathrm{S} / \mathrm{A}, \mathrm{Ds}, \mathrm{Dp}, \mathrm{Pt})$ químicos $(\mathrm{pH}$ em água e $\mathrm{KCl}, \Delta \mathrm{pH}$, $\left.\mathrm{Mg}^{2+}, \mathrm{Ca}^{2+}, \mathrm{K}^{+}, \mathrm{Na}^{+}, \mathrm{Al}^{3+}, \mathrm{H}^{+}+\mathrm{Al}^{3+}, \mathrm{P}, \mathrm{SB}, \mathrm{CTC}, \mathrm{V}, \mathrm{m}, \mathrm{MO}\right)$ nos horizontes diagnósticos superficiais e subsuperficiais

Em relação aos horizontes subsuperficiais, admitindo-se um corte na distância de ligação de 5,0 , observou-se a formação nítida de dois grupos semelhantemente ao que ocorreu no horizonte superficial, que individualizaram os segmentos de vertente: topo baixo, encosta e sopé de transporte e topo alto, correspondendo aos grupos G1 e G2, respectivamente (FIG. 6). Havendo diferenciação de apenas dois ambientes geomórficos, evidenciando assim as relações de interdependência entre os horizontes diagnósticos, corroborando com resultados encontrados por Campos et al., (2007) que estudou uma toposseqüência em Pereira Barreto, SP. De acordo com Young e Hammer (2000) técnicas estatísticas multivariadas (agrupamento) podem ser utilizadas para fins taxonômicos com sucesso, já que levam em consideração um conjunto de variáveis.

\section{Conclusões}

1. As variações do relevo favoreceram a presença de solos distróficos no topo alto e solos eutróficos no sopé de transporte;

2. Com o uso das técnicas estatísticas multivariadas (análise de agrupamento) foi possível distinguir diferentes ambientes geomórficos independentes dos segmentos de vertente identificados.

\section{Referências}

BARTHOLD, F. K. et al. Soil nutrient-landscape relationships in a lowland tropical rainforest in Panama. Forest Ecology and Management, v. 255, n. 03/04, p. 1135-1148, 2008.

BRASIL. Ministério das Minas e Energia. Projeto Radambrasil, folha SB. 20, Purus. Rio de Janeiro, 1978. 561 p.

CAMPOS, M. C. C. et al. Relações solo-paisagem em uma litosseqüência arenito-basalto na região de Pereira Barreto, SP. Revista Brasileira de Ciência do Solo, v. 31, n. 03, p. 519-529, 2007.

CAMPOS, M. C. C. Pedogeomorfologia aplicada à ambientes amazônicos do médio Rio Madeira. 2009. 242 f. Tese (Doutorado em Ciências do Solo) - Universidade Federal Rural de Pernambuco, Recife.

CARRÉ, F.; MCBRATNEY, A. B. Digital terron mapping. Geoderma. v. 128, n. 03/04, p. 340-353, 2005.

CENTRO DE PESQUISA DE RECURSOS MINERAIS (CPRM). Hidroclimatologia, geologia, recursos minerais, geomorfologia e unidades de paisagens. Manaus, 2001. 93 p. Relatório Técnico.

CUNHA, P. et al. Superfícies geomórficas e atributos de Latossolos em uma seqüência arenítico-basaltica da região de Jaboticabal (SP). Revista Brasileira de Ciência do Solo, v. 29, n. 01, p. 81-90, 2005.

DALRYMPLE, J. B. et al. A hipothetical nine unit land a surface model. Z. Geomorphology, v. 12, p. 60-76, 1968.

EMPRESA BRASILEIRA DE PESQUISA AGROPECUÁRIA (EMBRAPA). Centro Nacional de Pesquisa de Solos. Manual de métodos de análise de solo. Rio de Janeiro: Embrapa, 1997. 212 p.

GROBE, J. R. Aplicações da estatística multivariada na análise de resultados em experimentos com solos e animais. 2005. 145 f. Dissertação (Mestrado em Agronomia) - Universidade Federal do Paraná, Curitiba.

HAIR, J. F. et al. Análise multivariada de dados. Porto Alegre: Bookman, 2005. 593 p. 
HENDERSON, B. L. et al. Australia wide predictions of soil properties using decision trees. Geoderma. v. 124, n.03/04, p. 383-398, 2005.

INSTITUTO DE PROTEÇÃO AMBIENTAL DO ESTADO DO AMAZONAS (IPAAM) Zoneamento Ecológico Econômico do Sul-Sudeste do Amazonas. Amazonas: IPAAM, 2008. 53 p.

IPPOLITI, G. A. R. et al. Análise digital do terreno: ferramenta na identificação de pedoformas em microbacia na região de "Mar de Morros" (MG). Revista Brasileira de Ciência do Solo, v. 29, n. 02, p. 269-276, 2005.

GOMES, J. B. V. et al. Análise de componentes principais de atributos físicos, químicos e mineralógicos de solos do bioma cerrado. Revista Brasileira de Ciência do Solo, v. 28, n. 01, p. 137-153, 2004.

NUNES, W. A. G. A. et al. Relação solo-paisagem-material de origem e gênese de alguns solos no domínio do Mar de Morros, Minas Gerais. Revista Brasileira de Ciência do Solo, v. 25, n. 02, p. 341-354, 2001.

PACHEPSKY, Y. A. et al. Soil water retention as related to topographic variables. Soil Science Society American Journal, v. 65 , p. $1787-1795,2001$

PARK, S. J.; BURT, T. P. Identification and characterization of pedogeomorphological processes on a hillslope. Soil Science Society American Journal, v. 66, n. 06, p. 18971910, 2002.
SANTOS, R. D. et al. Manual de descrição e coleta de solos no campo. 5. ed. Viçosa. Sociedade Brasileira de Ciência do Solo, 2005. 100 p.

SOBIERAJ, J. A. H. et al. Spatial variability of soil hydraulic conductivity along a tropical rainforest catena. Geoderma, v. 108, p. 79-90, 2002.

STATISTICA. Statistica 7.0, EUA Software. Tucksa: StatSof, 2005.

VALLADARES, G. S. et al. Análise dos componentes principais e métodos multicritério ordinais no estudo de organossolos e solos afins. Revista Brasileira de Ciência do Solo, v. 32, p. 285-296, 2008.

VIDAL-TORRADO, P. et al. Pedogênese em uma seqüência Latossolo-Podzólico na borda de um platô na Depressão Periférica Paulista. Revista Brasileira de Ciência do Solo, v. 23, p. 909-921, 1999.

YEMEFACK, M.; ROSSITER, D. G.; NJOMGANG, R. Multiscale characterization of soil variability within an agricultural landscape mosaic system in southern Cameroon. Geoderma, v. 125, n. 01/02, p. 117-143, 2005.

YOEMANS, J. C.; BREMNER, J. M. A rapid and precise method for routine determination of organic carbon in soil. Communication Soil Science Plant Anal, v. 19, n. 13, p.1467-1476, 1988.

YOUNG, F. J.; HAMMER, R. D. Defining geographic soil bodies by landscape position, soil taxonomy, and cluster analysis. Soil Science Society American Journal, v. 64, p. 989-998, 2000. 